\title{
On the Complexity of Subfall Coloring of Graphs
}

\author{
Davi de Andrade ${ }^{1}$, Ana Silva ${ }^{1}$ \\ ${ }^{1}$ ParGO Group - Parallelism, Graphs and Optimization \\ Centro de Ciências - Departamento de Matemática \\ Universidade Federal do Ceará (UFC) \\ daviandradeiacono@gmail.com, anasilva@mat.ufc.br
}

\begin{abstract}
Given a graph $G$ and a proper $k$-coloring $f$ of $G, a$ b-vertex in $f$ is a vertex that is adjacent to every color class but its own. If every vertex is a b-vertex, then $f$ is a fall $k$-coloring; and $G$ has a subfall $k$-coloring if there is an induced $H \subseteq G$ such that $H$ has a fall $k$-coloring. The subfall chromatic number of $G$ is the largest positive integer $\psi_{f s}(G)$ such that $G$ has a subfall $\psi_{f s}(G)$-coloring. We prove that deciding whether a graph $G$ has a subfall $k$-coloring is NP-complete for $k \geq 4$, and characterize graphs having a subfall 3-coloring. This answers a question posed by Dunbar et al. (2000) in their seminal paper. We also prove polinomiality for chordal graphs and cographs, and present a comparison with other known coloring metrics based on heuristics.
\end{abstract}

\section{Introduction}

Given a simple graph $G$, a function $f: V(G) \rightarrow\{1, \cdots, k\}$ is a proper $k$-coloring of $G$ if $f(u) \neq f(v)$ for every $u v \in E(G)$; from now on, we call a proper coloring simply coloring. A classical problem of graph theory is to investigate the least value $k$ such that a graph $G$ has a $k$-coloring; this value is denoted by $\chi(G)$ and is called chromatic number. This is one of the hardest problems in Graph Theory, being NP-complete to decide whether a graph $G$ is 3-colorable even if $G$ is a 4-regular planar graph [Dailey 1980]. This is why it is natural to investigate colorings based on heuristics.

Given a graph $G$, a coloring $f$ of $G$ and a vertex $v \in V(G)$, we say that $v$ is a $b$-vertex in $f$ if it is adjacent to at least one vertex of each color class different from its own. Also, $f$ is a $b$-coloring if every color class has at least one b-vertex, and it is a fall coloring if every vertex is a b-vertex. The maximum value $k$ such that $G$ has a b-coloring (fall coloring) using $k$ colors is denoted by $b(G)\left(\psi_{f}(G)\right.$ ), and is called the $b$-chromatic (fall chromatic) number of $G$. We say that $G$ has a subfall $k$-coloring if there exists an induced subgraph $H \subseteq G$ such that $H$ has a fall $k$-coloring; the subfall chromatic number of $G$, denoted by $\psi_{f s}(G)$, is the largest value $k$ such that $G$ has a subfall $k$-coloring.

Fall and subfall colorings were introduced in [Dunbar et al. 2000], where among other things, they prove that the fall $k$-coloring problem is NP-complete. They then ask about the complexity of the subfall $k$-coloring and whether the inequality $\psi_{f s}(G) \leq b(G)$ holds. In this paper, we answer both questions. First, we prove that deciding whether a graph $G$ has a subfall $k$-coloring is NP-complete for $k \geq 4$, and give a structural characterization of graphs having a subfall 3-coloring. More specifically, we prove that $G$ has a subfall 3-coloring if and only if $G$ contains an induced cycle of length multiple of 3. We mention that a related problem, the complexity of finding an induced 
odd cycle, remained open for 14 years, and was only recently shown to be polynomial [Chudnovsky et al. 2020]. This hints towards polinomiality of subfall 3-coloring, which contrasts with fall 3-coloring, known to be NP-complete [Dunbar et al. 2000]. Regarding inequality $\psi_{f s}(G) \leq b(G)$, we answer their question in the negative, and also compare $\psi_{f s}(G)$ with other coloring parameters based on heuristics. Finally, we prove continuity of subfall colorings, i.e., that $G$ has a subfall $k$-coloring for every $k \in\left\{1, \cdots, \psi_{f s}(G)\right\}$, and we present a table comparing the complexity of the many parameters on chordal graphs, cographs and bipartite graphs, also filling two of those entries for subfall colorings, namely for chordal and cographs.

\section{Complexity of subfall $k$-coloring}

In this section, we present our complexity results. Note that every graph with non-empty edge set has a subfall coloring with 2 colors. So, we study the complexity of subfall $k$-coloring for $k \geq 3$. By applying a result in [Lauri and Mitillos 2019], we first obtain:

Theorem 1. Deciding whether a graph $G$ has a subfall $k$-coloring is NP-complete for every fixed $k \geq 4$.

For $k=3$, we could not settle the complexity of the problem, but we present the following characterization, that might help in its solution:

Theorem 2. A simple graph $G$ has subfall 3-coloring if and only if it contains a cycle $C_{3 k}$ as induced subgraph, for some positive integer $k$.

Given its NP-completeness for general graphs, it is natural to investigate the complexity of the problem restricted to specific graph classes. In this section, we investigate chordal graphs and cographs. For that, we need the concepts of perfect graphs and fall perfect graphs, the latter introduced in [Silva 2019]. The fall spectrum of $G$ is the set of values $k$ such that $G$ is fall $k$-colorable; it is denoted by $\mathcal{F}(G)$. A graph $G$ is perfect if, for every induced subgraph $H \subseteq G$, we have $\chi(H)=\omega(H)$; and is fall perfect if, for every induced subgraph $H \subseteq G$, we have $\mathcal{F}(H) \subseteq\{\chi(H)\}$. In [Silva 2019], the author proves that chordal graphs and cographs are fall perfect. Furthermore, chordal graphs and cographs are both hereditary subclasses of perfect graphs, i.e., if a graph $G$ is a chordal graph or is a cograph, then any induced subgraph of $G$ is also perfect. The aforementioned result and the following one give us that computing $\psi_{f_{s}}(G)$ is polynomial on chordal graphs and cographs, since computing $\omega(G)$ in these classes can be done in polynomial time.

Theorem 3. Let $\mathcal{G}$ be a class of graphs which are perfect and fall perfect. Then $\psi_{f s}(G)=$ $\omega(G)$, for every $G \in \mathcal{G}$.

\section{Continuity and other parameters}

In this section, we show one of the most important differences between fall coloring and subfall coloring: the continuity of the latter. In fact, there are graphs that do not even admit a fall coloring, which does not happen for subfall colorings. Moreover, there are graphs and values $k, m$ and $n$, with $k<m<n$, that admit fall $k$-coloring and fall $n$ coloring, but do not admit fall $m$-coloring, which shows the discontinuity of fall coloring of graphs. See Figure 1 for an example.

Proposition 4. Let $G$ be a graph; then $G$ has a subfall $k$-coloring, for every $k \in\left[\psi_{f_{s}}(G)\right]$. 

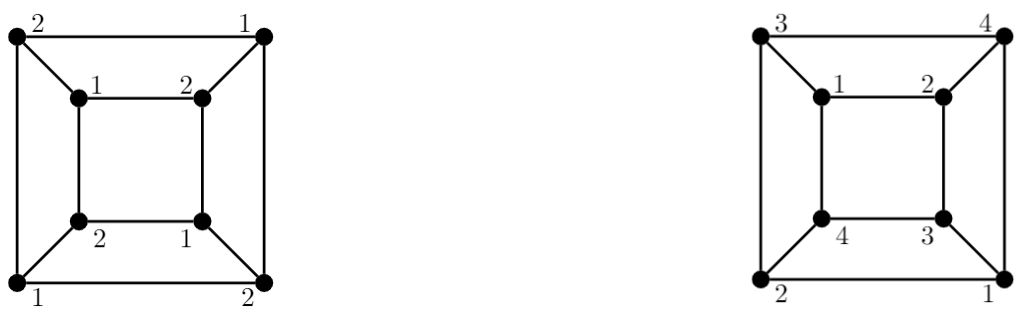

Figure 1. Graph that has fall 2-coloring and fall 4-coloring, but no fall 3-coloring.

We now give some further definitions. Let $f$ be a coloring of $G$. We say that $v$ is a Grundy vertex of color $i$ in $f$ if $f(v)=i$ and $v$ is adjacent to at least one vertex in color class $j$ for each $1 \leq j<i$. Additionally, $f$ is a Grundy $k$-coloring if it is a $k$-coloring and each vertex $v \in V(G)$ is a Grundy vertex; the maximum value $k$ such that $G$ has a Grundy $k$-coloring is called Grundy number and denoted by $\Gamma(G)$. A natural concept of graph theory is to investigate possible relations between graph parameters. It is folklore knowledge that $\psi_{f}(G) \leq b(G)$, that $\psi_{f}(G) \leq \Gamma(G)$, and that $b(G)$ and $\Gamma(G)$ are not related. Below, we analyze the relation between each of the aforementioned parameters and $\psi_{f s}(G)$ adding the inequality $\psi_{f}(G) \leq \psi_{f s}(G)$, established in [Dunbar et al. 2000].

Proposition 5. The following statements are true:

a. There exists $G_{1}$ such that $\psi_{f s}\left(G_{1}\right)<\chi\left(G_{1}\right)$. Additionally, for every positive integer $k$, there exists $G_{2}$ such that $\psi_{f s}\left(G_{2}\right)-\chi\left(G_{2}\right)=k$;

$b$. For each positive integer $k$, there exist graphs $G_{1}$ and $G_{2}$ such that: $b\left(G_{1}\right)-$ $\psi_{f s}\left(G_{1}\right)=\psi_{f s}\left(G_{2}\right)-b\left(G_{2}\right)=k$;

c. For every graph $G$, we have $\psi_{f}(G) \leq \psi_{f s}(G) \leq \Gamma(G)$.

In order to establish arbitrarily high $\chi(G)-\psi_{f s}(G)$, it is natural to look for a graph $G$ with arbitrarily large $\chi(G)$ and such that $\psi_{f_{s}}(G)=2$. By Theorem 2, such a graph must be $C_{3 n}$-free for every $n$. This seems to be very challenging since a $C_{3}$-free $G$ with arbitrarily large $\chi(G)$ was not known until 1954 when the first construction was given by [Descartes 1954], and constructions forcing high girth appeared only around 10 years after Erdős presented his groundbreaking probabilistic proof in 1959.

\section{Final Remarks}

As shown in the previous sections, the NP-completeness of subfall coloring leads us to investigate the complexity for restricted graph classes; we have chosen to investigate bipartite graphs, chordal graphs and cographs, which are between the most investigated graph classes. It is also natural to be interested in comparing the complexity of the previously defined problems on these classes. Table 1 summarizes the known results. The problem marked with "?" is still open.

\begin{tabular}{|c|c|c|c|}
\hline & bipartite & chordal & cograph \\
\hline fall coloring & NP-complete & NP-complete & Polynomial \\
\hline subfall coloring & $?$ & Polynomial* & Polynomial* \\
\hline b-coloring & NP-complete & NP-complete & Polynomial \\
\hline Grundy & NP-complete & NP-complete & Polynomial \\
\hline
\end{tabular}

Table 1. The entries marked with * follow from Theorem 3. 
The proof of NP-completeness of fall colorings on bipartite graphs is given in [Dunbar et al. 2000], while in [Silva 2019] the author proves NP-completeness for chordal graphs and polynomiality for cographs. As for b-coloring, the NPcompleteness for bipartite and chordal graphs were proved in [Irving and Manlove 1999] and [Havet et al. 2012] respectively, while polynomiality for cographs is given in [Bonomo et al. 2009]. Finally, Grundy coloring is NP-complete on bipartite graphs [Havet and Rocha 2013] and on chordal graphs [Sampaio 2012], and polynomial on cographs [Araujo and Linhares Sales 2012]. We then notice that all of these problems behave similarly, with a deviation only for subfall coloring of chordal graphs.

\section{References}

Araujo, J. and Linhares Sales, C. (2012). On the Grundy number of graphs with few $P_{4}$ 's. Discrete Applied Mathematics, 160(18):2514-2522.

Bonomo, F., Duran, G., Maffray, F., Marenco, J., and Valencia-Pabon, M. (2009). On the b-Coloring of Cographs and $P_{4}$-Sparse Graphs. Graphs and Combinatorics, 25:153167.

Chudnovsky, M., Scott, A., Seymour, P., and Spirkl, S. (2020). Detecting an Odd Hole. J. $A C M, 67(1)$.

Dailey, D. P. (1980). Uniqueness of colorability and colorability of planar 4-regular graphs are NP-complete. Discrete Mathematics, 30(3):289-293.

Descartes, B. (1954). Solution to advanced problem no. 4526. Amer. Math. Monthly, $61: 352$.

Dunbar, J., Hedetniemi, S., Hedetniemi, S., Jacobs, D., Knisely, J., Laskar, R., and Rall, D. (2000). Fall Colorings of Graphs. J. of Combinatorial Mathematics and Combinatorial Computing, 33:257-273.

Havet, F., Linhares Sales, C., and Sampaio, L. (2012). b-coloring of tight graphs. Discrete Applied Mathematics, 160(18):2709-2715.

Havet, F. and Rocha, L. (2013). On the Grundy and b-Chromatic Numbers of a Graph. Algorithmica, 65:885-899.

Irving, R. W. and Manlove, D. F. (1999). The b-chromatic number of a graph. Discrete Applied Mathematics, 91(1):127-141.

Lauri, J. and Mitillos, C. (2019). Complexity of Fall Coloring for Restricted Graph Classes. 11638:352-364.

Sampaio, L. (2012). Algorithmic aspects of graph colourings heuristics. PhD thesis, Université Nice Sophia Antipolis.

Silva, A. (2019). Graphs with small fall-spectrum. Discrete Applied Mathematics, 254:183-188. 